\title{
Marine bacteriophage reproduction under nutrient-limited growth of host bacteria. II. Investigations with phage-host system [H3:H3/1]
}

\author{
Karlheinz Moebus
}

Biologische Anstalt Helgoland (Meeresstation), D-27498 Helgoland, Germany

\begin{abstract}
When host bacterium $\mathrm{H} 3$ is cultivated in artificial seawater at a concentration of $0.6 \mathrm{mg}$ organic nutrients $\mathrm{ml}^{-1}$, its reaction to infection by phage $\mathrm{H} 3 / 1$ changes dramatically with the duration of incubation before phage attack. Cells infected when still in their logarithmic growth phase rapidly produce progeny phage until breakdown of the phage-sensitive population. For cells infected after entering the stationary phase, rate and extent of phage propagation by resting cells decreases for some time, but both parameters rather suddenly increase again for cells infected after prolonged incubation. Phage production may then reach the same level attained by cells infected during logarithmic growth phase; atthough, with increasing phage concentration, resting cells very effectively become protected from phage attack by pseudolysogeny. This course of events is mainly influenced by the method of incubation of the host (shaken vs still), by the age of the cells before infection, and presumedly by changes in physiological traits of the bacteria when serially subcultured for extended periods of time. The reduction in volume of bacterial culture by repeated withdrawal of aliquots was found to be of minor importance; however, phage production was measurably affected by the transfer of cells to fresh receptacles. With cells which entered their stationary phase up to 36 h before infection, an initial phage concentration of between 10 and $10^{3} \mathrm{PFU}$ (plaque forming units) $\mathrm{ml}^{-1}$ was found to determine the extent, but not the rate, of phage production. The aforementioned observations were also made with cells starved for 3,8 , and 22 additional days before inoculation, except with initial PFU concentrations of 10 and $10^{2} \mathrm{PFU} \mathrm{m} \mathrm{m}^{-1}$, when an appreciable increase in phage production was found. In cell suspensions seeded with phage $48 \mathrm{~d}$ after set up of the experiment, the highest phage production was found with the lowest initial phage concentration and vice versa. This finding, i.e. the inverse relationship between production and initial concentration, is not in agreement with any of the current hypotheses concerning bacteriophage ecology.
\end{abstract}

KEY WORDS: Virus Phage Bacteria Reproduction

\section{INTRODUCTION}

In the preceding paper the results of an investigation performed with 6 marine phage-host systems (PHS) were presented (Moebus 1996 in this issue). During that study, experiments were conducted with samples enriched to $0.6 \mathrm{mg}$ organic nutrients $\mathrm{ml}^{-1}$, and several observations were made which called for a more detailed investigation.

For example, it was observed that, after some time of nutrient deficiency, host bacterium $\mathrm{H} 40$ reacted to transfer to fresh receptacles with an increase in the number of colony forming units (CFU). Is this reaction unique to $\mathrm{H} 40$, and, if not, does it influence the cells' reaction to infection by phage?

With host $\mathrm{H} 3$ incubated under ongoing conditions of starvation it was found that, after a period of decreasing efficiency in phage production, cells were able to produce phage at an increased rate and to an extent corresponding with that observed for cells infected before starvation. Was this observation a single event or would it be reproducible? Strong indications were also found that $\mathrm{H} 3$ develops pseudolysogeny (Hayes 1964, Baess 1971) as a means of survival in the presence of a high concentration of infective phage particles. 
Finally, the importance of bacteriophage concentration for phage propagation needs to be addressed. In the aforementioned investigation by Moebus (1996), $10^{3}$ infective virions $\mathrm{ml}^{-1}$ was the lowest concentration of plaque forming units (PFU) used. According to the majority of publications dealing with PFU concentration in seawater (for review see Moebus 1987), this is an extraordinarily high concentration of infectious phage relative to a specific bacterial host.

Experiments performed to shed some light on these problems are presented in this paper

\section{MATERIAL AND METHODS}

Essentially, the same materials and methods as those given by Moebus (1996) were employed, with the following exceptions: (1) The phage-host system [H3:H3/1.] and synthetic seawater with yeast extract pius pepione (1:5 parts, $Y E P$ ) at $0.6 \mathrm{mg} !^{-i}$ were used throughout. (2) The test for pseudolysogeny was extended. It will be described in the text with its results. (3) Acridine-orange direct counts were made several times during incubation of batch cultures and/or at the end of investigation of samples (4) Unless stated otherwise, all incubations were as still culture at $20^{\circ} \mathrm{C}$.

To test for delayed phage release by starved cells, YEP was added during the course of the experiments. The necessary amounts of properly diluted YEP solution were calculated from weights determined for the empty and filled receptacles as well as the volume of aliquots withdrawn for CFU and PFU titrations. Distilled water was added to compensate for loss by evaporation.

The term batch is used throughout for phage-free volumes of 100 or $200 \mathrm{ml}$, while the term sample refers to aliquots of 18 or $20 \mathrm{ml}$ withdrawn from batches and either inoculated with phage or used as controls. Information concerning length of time refers to the time elapsed since the set up of a batch; otherwise, the interval after phage addition is explicitly stated.

\section{RESULTS}

\section{Reproduction of phage $\mathrm{H} 3 / 1$ by its host $\mathrm{H} 3$}

For samples withdrawn and seeded 2 to $14 \mathrm{~d}$ after set up of the batch, it was found (Moebus 1996) that the ability of $\mathrm{H} 3$ to reproduce phage greatly decreased relative to that of $\mathrm{H} 3$ in samples seeded within the first 2 d. For samples inoculated after Day 14, phage production increased, at first with a lag of 1 to $2 \mathrm{~d}$ following sample transfer, some days later without the lag.
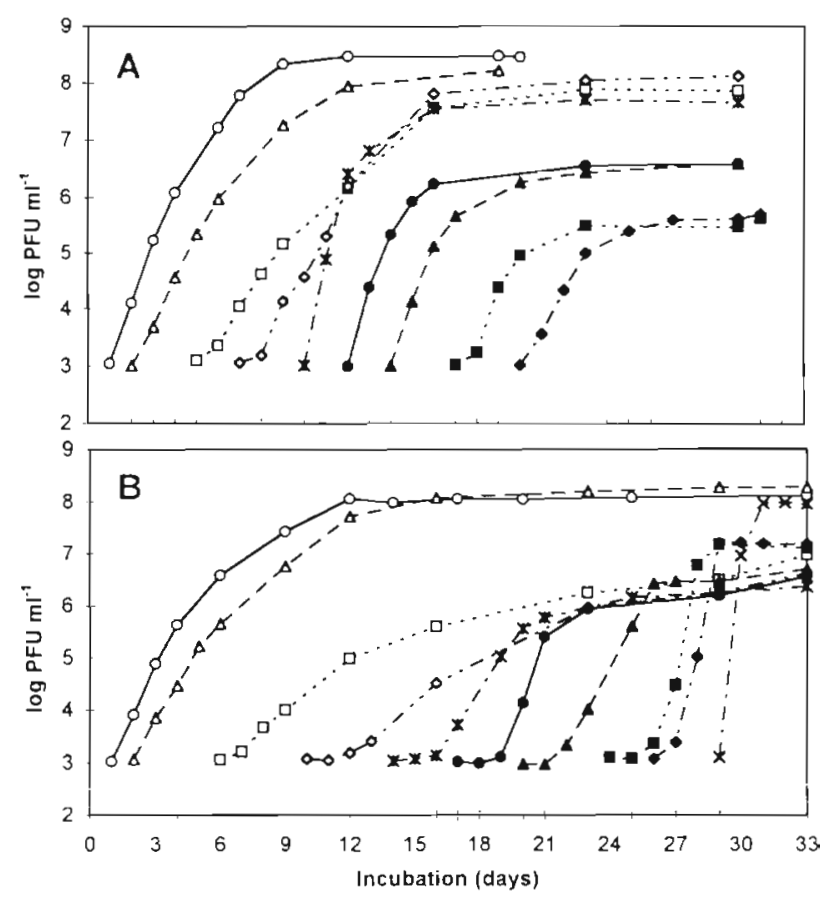

Fig. 1 Denendence of reproduction of phage $13 / 1$ by host $H 3$ on duration and type of incubation prior to withdrawal and inoculation of samples ( $18 \mathrm{ml}$ each) with phage. $200 \mathrm{~m}$ ) batch cultures in synthetic seawater with $0.6 \mathrm{mg}$ organic nutrients $l^{-1}$ incubated $(A)$ shaken and $(B)$ as still culture

Then, rate and extent of phage production was almost the same as that observed with cells infected shortly before the end of their logarithmic growth phase (i.e. within the first $2 \mathrm{~d}$ ).

To test for reproducibility of this phenomenon and its possible dependence on the physiological state of the cells, an experiment was undertaken with 4 batch cultures of $200 \mathrm{ml}$ each. Of these 4 batches, 2 were incubated shaken (160 strokes $\mathrm{min}^{-1}$ ) and 2 as still culture. Samples $(18 \mathrm{ml})$ were collected (and inoculated with about $10^{3} \mathrm{PFU} \mathrm{ml}^{-1}$ ) at different times between 1 and $20 \mathrm{~d}$ after the start of the experiment from the shaken batches, and between 1 and $29 \mathrm{~d}$ from the unshaken batches. All samples were then incubated as still cul.ture.

The outcome of this experiment confirms that the more or less sudden restoration of highly effective phage reproduction is a reproducible phenomenon. (Fig 1). It occurred earlier in samples from shaken batches than in those from still batches. With samples taken from the shaken batches, the relative increase in phage production lasted for only a short time and was followed by a resumed decrease. The maximal extent of phage reproduction was independent of the mode of incubation; however, considerable differences between samples collected at the same time from parallel batches were found 
Table 1 Production of phage H3/1 by host H3. Comparative description of shape of PFU concentration curves, as well as final PFU concentrations $\left(\mathrm{ml}^{-1}\right\}$, found for samples from batches parallel to those for which results are shown in Fig. 1. Concrntrations in $\mathrm{ml}^{-1}$ Host $\mathrm{H} 3$ grown in $200 \mathrm{ml}$ batches of synthetic seawater with $0.6 \mathrm{mg}$ organic nutrients $\mathrm{l}^{-1}$ Striking observations are in italics

\begin{tabular}{|c|c|c|c|c|}
\hline Sample & Shape of curve as compared to Fig. 1 & Day & PFU & $\mathrm{PFU}_{\mathrm{Fig}}$ : \\
\hline \multicolumn{5}{|c|}{ A. Batch incubated shaken } \\
\hline $3 \mathrm{~d}$ & Similar to 2 d sample, less steep & 19 & $4.2 \times 10^{7}$ & $1.7 \times 10^{8}$ \\
\hline $5 d$ & Much the same as 6 d sample from unshaken batch shown in Fig. 1 & 30 & $6.1 \times 10^{6}$ & $7.2 \times 10^{7}$ \\
\hline $8 d$ & Slight lag followed by straight increase for $7 \mathrm{~d}$ to $10^{7}$ & 30 & $5.9 \times 10^{7}$ & $1.3 \times 10^{8}$ \\
\hline $10 \mathrm{~d}$ & $1 \mathrm{~d}$ lag followed by less steep increase & 30 & $6.3 \times 10^{7}$ & $4.5 \times 10^{7}$ \\
\hline $11 \mathrm{~d}$ & Parallel to $10 \mathrm{~d}$ sample of this batch & 30 & $1.6 \times 10^{7}$ & $3.9 \times 10^{6}$ \\
\hline $14 \mathrm{~d}$ & Similar & 30 & $4.3 \times 10^{6}$ & $3.9 \times 10^{6}$ \\
\hline $17 \mathrm{~d}$ & Similar & 31 & $2.5 \times 10^{6}$ & $4.2 \times 10^{5}$ \\
\hline $20 \mathrm{~d}$ & Similar & 31 & $1.6 \times 10^{8}$ & $5.0 \times 10^{5}$ \\
\hline \multicolumn{5}{|c|}{ B. Batch incubated as still culture } \\
\hline $3 d$ & After slight lag similar to $2 \mathrm{~d}$ sample from other batch & 33 & $5.9 \times 10^{7}$ & $1.9 \times 10^{8}$ \\
\hline $6 \mathrm{~d}$ & Same until Day $29: 1.7 \times 10^{6}$ vs $1.6 \times 10^{6}$ & 33 & $2.7 \times 10^{6}$ & $9.1 \times 10^{6}$ \\
\hline $11 \mathrm{~d}$ & Steeper increase than in $10 \mathrm{~d}$ sample from other batch & 33 & $1.1 \times 10^{8}$ & $4.8 \times 10^{6}$ \\
\hline $14 \mathrm{~d}$ & Steeper increase until Day $20\left(4.5 \times 10^{6}\right)$ & 33 & $1.7 \times 10^{7}$ & $2.3 \times 10^{\mathrm{b}}$ \\
\hline $17 \mathrm{~d}$ & $\begin{array}{l}2 \mathrm{~d} \text { lag followed by } 3 \mathrm{~d} \text { of steeper increase to } 2.2 \times 10^{h} \text { vs } 8.7 \times 10^{5} \text { in } \\
\text { sample from other batch }\end{array}$ & 33 & $1.3 \times 10^{7}$ & $3.5 \times 10^{6}$ \\
\hline $20 \mathrm{~d}$ & Similar & 33 & $1.0 \times 10^{7}$ & $4.9 \times 10^{b}$ \\
\hline $24 \mathrm{~d}$ & Almost no lag, $P F U_{\max }$ reached after $3 d$ & 33 & $3.3 \times 10^{7}$ & $1.3 \times 10^{7}$ \\
\hline $26 d$ & No lag, $P F U_{\max }$ reached after $3 d$ & 33 & $6.1 \times 10^{7}$ & $1.5 \times 10^{7}$ \\
\hline $29 \mathrm{~d}$ & No lag, but much less steep: after 1 d $2.4 \times 10^{4}$ vs $6.0 \times 10^{\text {h }}$ & 33 & $6.6 \times 10^{11}$ & $8.9 \times 10^{7}$ \\
\hline
\end{tabular}

Fig 1 presents PFU results found for 1 of each of the differently incubated batches. In Table 1, the phage production curves for the samples from the other two respective batches are compared with those shown in Fig. 1. The most blatant differences are underlined.

CFU results for the 4 batches were more or less the same for all but the samples withdrawn on the first day: following phage addition, CFU concentrations generally gradually decreased from over $10^{6} \mathrm{ml}^{-1}$ to between $10^{5}$ and $2 \times 10^{4} \mathrm{CFU} \mathrm{ml}^{-1}$.

Table 2 shows selected data for the 4 samples collected after $1 \mathrm{~d}$, including CFU counts. Note that in the sample from Batch 4 the final PFU titre was attained after only $4 d$ and that the first CFU minimum observed 1 d later was nonetheless comparatively high. It was immediately followed by a return to the CFU level of the batch culture, i.e. to $1.7 \times$ $10^{6} \mathrm{PFU} \mathrm{m} \mathrm{m}^{-1}$. Further decline in CFU numbers of this sample was similar to that in the batch culture.

During the course of the experiments the volume of each batch decreased with each sample withdrawal. This may have triggered the increase in efficiency of phage production as observed after prolonged incubation. To check this possibility, an experiment was started with four
$200 \mathrm{ml}$ batches as well as 3 single batches containing 164, 128, and $92 \mathrm{ml}$ respectively. The first samples were collected from the 3 smaller batches when the $3 \mathrm{rd}, 5$ th, and 7 th samples, respectively, were withdrawn from 3 of the $200 \mathrm{ml}$ batches.

The same three $200 \mathrm{ml}$ batches were employed to further test the reproducibility of results as well as the influence of added nutrients on phage production. With the fourth $200 \mathrm{ml}$ batch, an initial test was carried out to find out to what degree the transfer of samples into fresh receptacles may influence phage replication.

The outcome of this experiment was gravely influenced by the bacterial population employed. For unknown reason(s) it tended to produce flakes to a

Table 2. PFU and CFU concentrations $\left(\mathrm{ml}^{-1}\right)$ in the first samples (withdrawn after 1 d) from each of 4 batches incubated as either still culture ( 1 and 2) or shaken ( 3 and 4 ). Host $\mathrm{H} 3$ in synthetic seawater with $0.6 \mathrm{mg}$ organic nutrients $\mathrm{l}^{-1}$ PFU: maximal and final concentrations. CFU: first and second minima (note that second minima are also final concentrations). First samples from shaken batches were used only until Day 19 of the experiment

\begin{tabular}{|rrrrrrrrr|}
\hline Batch & PFU & Day & PFU & Day & $\begin{array}{c}\text { CFU } \\
1 \text { st min. }\end{array}$ & Day & $\begin{array}{c}\text { CFU } \\
\text { 2nd min. }\end{array}$ & Day \\
\hline 1 & $1.2 \times 10^{8}$ & 12 & $1.3 \times 10^{8}$ & 33 & $4.2 \times 10^{3}$ & 9 & $1.5 \times 10^{4}$ & 33 \\
2 & $7.4 \times 10^{7}$ & 12 & $2.7 \times 10^{8}$ & 33 & $7.2 \times 10^{3}$ & 17 & $4.6 \times 10^{3}$ & 33 \\
3 & $1.2 \times 10^{6}$ & 4 & $3.0 \times 10^{8}$ & 19 & $1.4 \times 10^{3}$ & 9 & $2.5 \times 10^{4}$ & 19 \\
4 & $7.0 \times 10^{7}$ & 4 & $7.1 \times 10^{7}$ & 19 & $8.9 \times 10^{5}$ & 5 & $8.3 \times 10^{4}$ & 19 \\
\hline
\end{tabular}


degree never observed before or afterwards. This made CFU determinations almost worthless. Curves constructed from the results zigzagged wildly. In a number of cases the CFU counts from parallel plates differed by $500 \%$ or more. Trends in overall CFU reductions, however, were the same as in earlier experiments. With the duration of the experiment, the importance of flakes abviously decreased. This is indicated by results of direct counts, increasingly reliable CFU determinations, and observations made in regard to phage production. (During previously performed experiments, weak flake formation was observed only some time after phage addition to samples, but never with batches.)

Fig. 2 presents selected data from this experiment. Curves with filled symbols are constructed from mean values of PFU concentrations determined for the 3 parallel $200 \mathrm{ml}$ batches. Open symbols represent the smaller batches.

Comparatively limited decline in rate and extent of phage production was observed in samples from the three $200 \mathrm{ml}$ batches. Maximal concentrations of less than $5 \times 10^{5}$ PFU ml $\mathrm{m}^{-1}$ were found in only 4 out of 30 samples. The increase to signuficantly higher PFU production after prolonged incubation was observed in samples from only 1 of the 3 batches. It occurred in samples withdrawn after 31,35 , and $40 \mathrm{~d}$ of batch culture. This is considerably later than the 24 to $29 \mathrm{~d}$ samples for which it occurred in the aforementioned experiment.

Findings obtained with the 164 and $128 \mathrm{ml}$ batches very much resemble those found with the $200 \mathrm{ml}$ batches. There was no appreciable reduction in phage reproduction and only unspectacular recovery. The latter, however, occurred in samples collected after 31 ,
35, and $40 \mathrm{~d}$, as for samples from the exceptional $200 \mathrm{ml}$ batch reported above.

Only 4 samples could be withdrawn from the $92 \mathrm{ml}$ batch. Results for the first 3 samples resembled those found for other samples from other batches in this experiment. Near to maximal PFU concentration, rang-

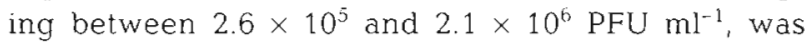
observed in these 3 samples after 19,18 , and $18 \mathrm{~d}$ following phage addition. With the fourth sample (taken on Day 40), however, $3.5 \times 10^{6}$ PFU ml-1 was found after only $2 \mathrm{~d}$, and the final PFU concentration of almost $10^{8}$ infective particles $\mathrm{ml}^{-1}$ was the highest ever observed in the present experiment without additional YEP. For CFU counts, the least (but still) unsatisfactory results were found during this experiment with the $92 \mathrm{ml}$ batch.

The 3 identically treated $200 \mathrm{ml}$ batches were also used to test the influence of nutrients added when the maximal PFU concentration had been (almost) stcady for a few days. YEP was then added to establish a concentration of $0.6 \mathrm{mg}$ fresh YEP ${ }^{-1}$

In the few samples with the lower maximal. PFU concentrations referred to above, a conspicuously steep increase in PFU titre following YEP addition was found. In sum, however, the results do not indicate that release of mature phage in significant portions was triggered by newly increased metabolic activity. After YEP addition in all tested samples, the PFU concentration levelled off at $10^{8} \mathrm{PFU} \mathrm{ml^{-1 }}$

The fourth $200 \mathrm{ml}$ batch was used in 2 intervals (Fig. 3), beginning after $52 \mathrm{~d}$ of incubation without any movement whatsoever. On Day 52, CFU determinations were made prior to and after vigorous shaking, and four $18 \mathrm{ml}$ samples were withdrawn on Day 53. Phage was added to a different one of these samples

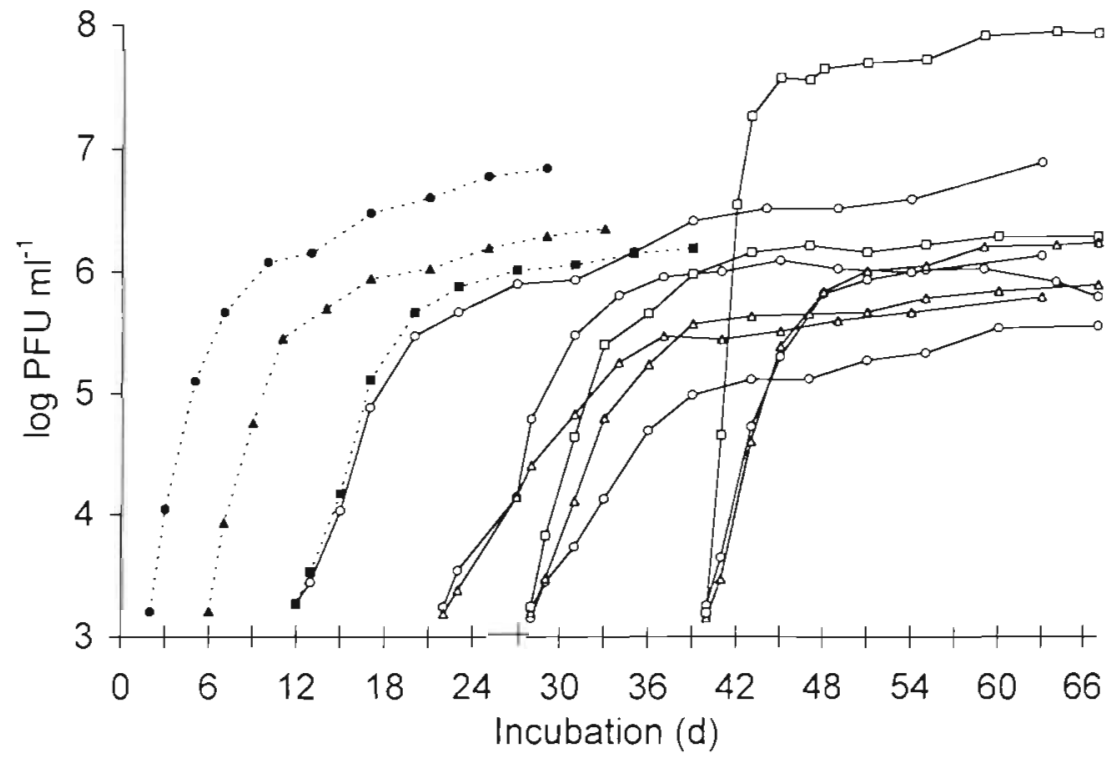

Fig. 2. Dependence of reproduction of phage $\mathrm{H} 3 / 1$ by host $\mathrm{H} 3$ on batch size and duration of incubation as still culture before withdrawal and inoculation of samples $(18 \mathrm{ml})$ with phage (selected results are shown). Filled symbols. mean values for samples from 3 parallel $200 \mathrm{ml}$ batch cultures. Other batch sizes: $164 \mathrm{ml}(0), 128 \mathrm{ml}(\Delta)$, and $92 \mathrm{ml}$ (b). Host H3 grown in synthetic seawater with $0.6 \mathrm{mg}$ organic nutrients $\mathrm{I}^{-1}$ 
Fig. 3. Reproduction of phage $\mathrm{H} 3 / 1$ by host $\mathrm{H} 3$ as influenced by sample transfer into fresh receptacles and the time lag before seeding with phage. Multiple samples $(18 \mathrm{ml})$ were withdrawn from a $200 \mathrm{ml}$ batch culture (still culture) in synthetic seawater with $0.6 \mathrm{mg}$ organic nutrients $\mathrm{l}^{-1}$ after 53 and $85 \mathrm{~d}$ and inoculated with phage at varying times thereafter

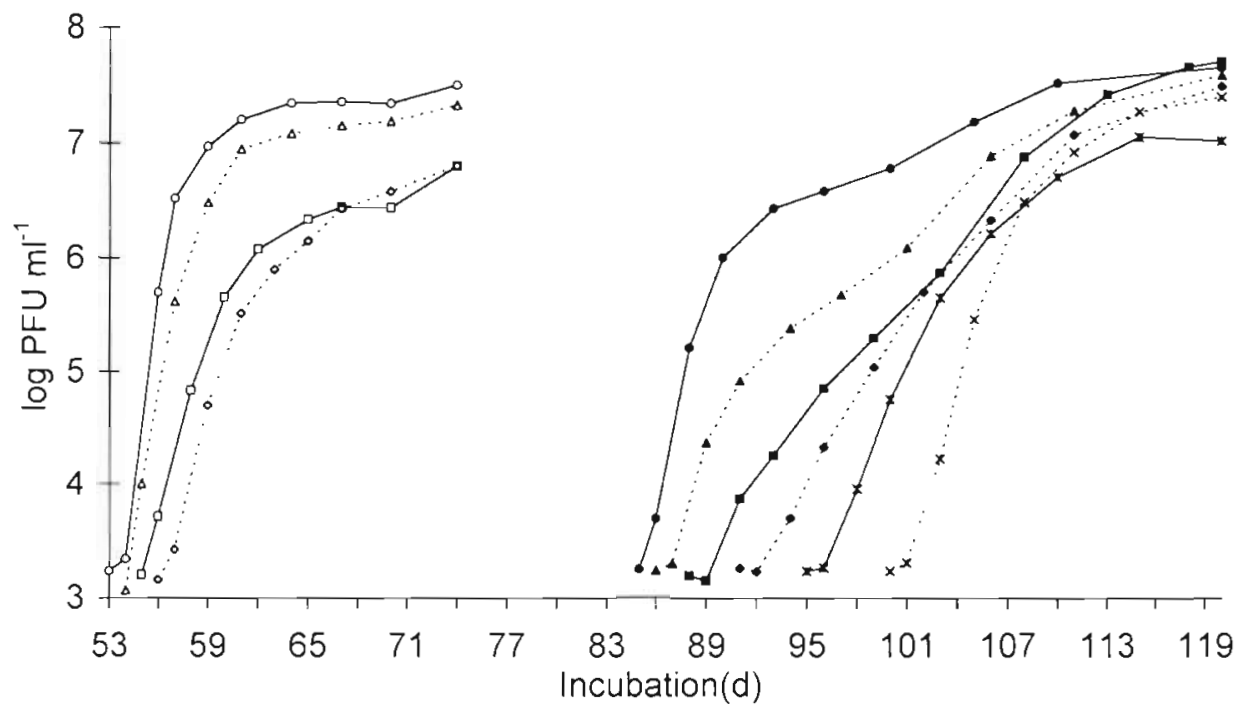

from Day 53 through Day 56. Propagation of phage was followed until Day 74. Final concentrations of between $5 \times 10^{6}$ and $3 \times 10^{7} \mathrm{PFU} \mathrm{m} \mathrm{m}^{-1}$ were found for the 4 samples, with time after phage addition and PFU concentration directly correlated. The strongest, though still weak, initial slow-down in phage production was observed with the sample seeded on Day 56.

The second interval was started on Day 85 of the experiment, with 6 samples collected at that time. They were inoculated with phage $0,1,3,6,10$, and $15 \mathrm{~d}$ thereafter (i.e. Days 85,86, 88, 91,95 and 100). At the end of this interval, PFU concentrations in the 6 samples ranged between $10^{7}$ and $5 \times 10^{7} \mathrm{PFU} \mathrm{m} \mathrm{m}^{-1}$. There was a significant reduction in the rate of phage production in the samples seeded with phage 1 and $3 \mathrm{~d}$ after collection. However, starting with the sample inoculated $6 \mathrm{~d}$ after withdrawal, the rate began to increase again. In the sample inoculated $15 \mathrm{~d}$ after collection, the initial rate of phage production was reestablished.

The results obtained with both series of samples indicate that phage reproduction is discernibly influenced by the transfer of samples into fresh receptacles.

Final experiments were concerned with (1) the influence of initial phage concentration on phage reproduction and (2) the variation in the course of phage production when sample transfer is avoided.

To study the influence of initial phage titre, three $200 \mathrm{ml}$ batches were prepared and incubated as still culture. Six $18 \mathrm{ml}$ samples were alternately collected from Batches 1, 2, and
3 after 1,3, and 6 , and 12,24, and $48 \mathrm{~d}$ of incubation (i.e. 6 samples from Batch 1 on Days 1 and 12, from Batch 2 on Days 3 and 24, etc.). Immediately after withdrawal, phage was added to attain initial PFU concentrations of about $10^{3}$ ( 1 sample), $10^{2}$ (2 samples), and $10 \mathrm{ml}^{-1}$ (3 samples). Phage reproduction and bacterial survival were followed for $3 \mathrm{wk}$. During this experiment, all CFU determinations were of the usual reliability. This is in strong contrast to the aforementioned experiment in which heavy flaking occurred, although the bacteria used in this experiment were direct descendants of those used in the other experiment.

Table 3 presents the final PFU concentrations found in the samples $21 \mathrm{~d}$ after phage addition. Fig. 4 shows the findings for samples collected from Batches 1, 2, and 3 , after 1,24 , and $48 \mathrm{~d}$ of incubation, respectively.

With an initial PFU of $10^{3} \mathrm{ml}^{-1}$ present, observations were similar to those already described: a decrease in rate and extent of phage production was followed by

Table 3. Final PFU concentrations in samples inoculated to different initial PFU concentrations. Host $\mathrm{H} 3$ in $200 \mathrm{ml}$ batches in synthetic seawater with $0.6 \mathrm{mg}$ organic nutrients $1^{-1}$ Initial titre of phage $\mathrm{H} 3 / 1$ was about $10^{3}$ ( 1 sample), $10^{2}$ (2 samples), and 10 infecting particles $\mathrm{ml}^{-1}$ (3 samples). Batch:Day shows batch number and day of sample withdrawal/inoculation. Values within groups of samples are arranged in ascending order. Extreme values are in italics

\begin{tabular}{|c|c|c|c|c|c|}
\hline \multirow[t]{2}{*}{ Batch:Day } & \multicolumn{5}{|c|}{ Initial PFU concentration $\mathrm{ml}^{-1}$} \\
\hline & $10^{3}$ & $10^{2}$ & & 10 & \\
\hline $1: 1$ & $6.2 \times 10^{6}$ & $5.3 \times 10^{5} 5.6 \times 10^{5}$ & $1.1 \times 10^{4}$ & $3.5 \times 10^{4}$ & $6.9 \times 10^{4}$ \\
\hline $2: 3$ & $2.5 \times 10^{6}$ & $8.0 \times 10^{4} \quad 1.4 \times 10^{5}$ & $2.2 \times 10^{3}$ & $4.8 \times 10^{3}$ & $9.9 \times 10^{3}$ \\
\hline $3: 6$ & $3.4 \times 10$ & $3.2 \times 10^{5} \quad 9.8 \times 10^{12}$ & $5.4 \times 10^{2}$ & $5.6 \times 10^{2}$ & $2.7 \times 10^{4}$ \\
\hline $1: 12$ & $1.8 \times 10^{6}$ & $5.3 \times 10^{3} \quad 1.8 \times 10^{3}$ & $2.2 \times 10^{2}$ & $2.3 \times 10^{2}$ & $5.8 \times 10^{5}$ \\
\hline $2: 24$ & $1.8 \times 10^{j}$ & $1.4 \times 10^{3} 4.6 \times 10^{4}$ & $3.1 \times 10^{3}$ & $1.8 \times 10^{4}$ & $5.6 \times 10^{6}$ \\
\hline $3: 48$ & $4.7 \times 10^{5}$ & $4.3 \times 10^{5} \quad 5.5 \times 10^{5}$ & $1.4 \times 10^{n}$ & $1.9 \times 10^{6}$ & $2.4 \times 10^{6}$ \\
\hline
\end{tabular}




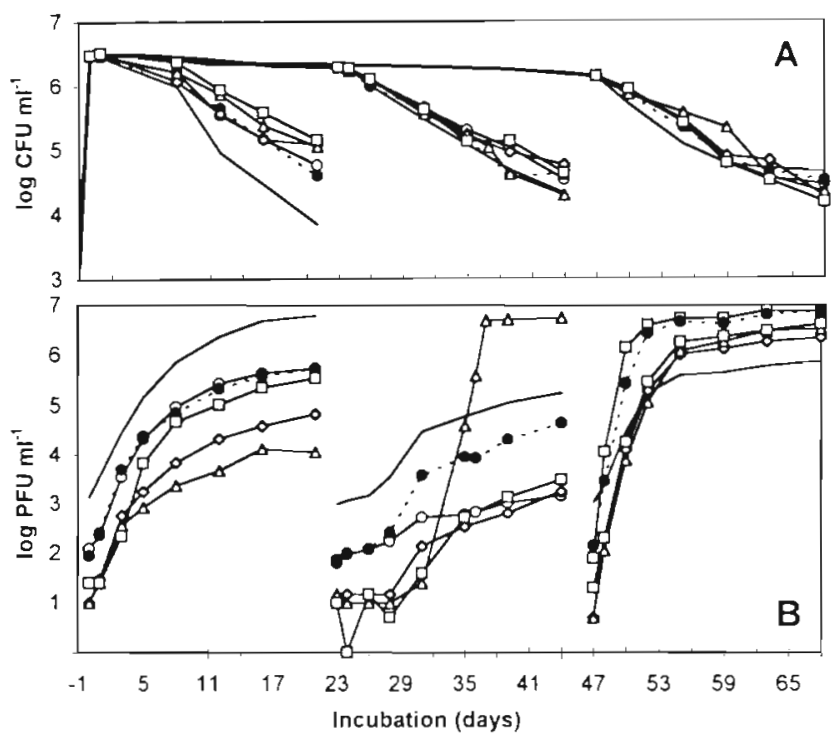

Fig. 4. Dependence of reproduction of phage H3/1 by host $\mathrm{H} 3$ on time of sample collection and inutial concentration of phage. Data shown are for groups of six $18 \mathrm{ml}$ samples collected from three $200 \mathrm{ml}$ batch cultures of $\mathrm{H} 3$ in synthetic seawater with $0.6 \mathrm{mg}$ organic nutrients $\mathrm{l}^{-1}$ after $1 \mathrm{~d}$ (Batch 1), $24 \mathrm{~d}$ (Batch 2) and $48 \mathrm{~d}$ (Batch 3) of incubation without agitation Initial phage concentrations were $10^{3}$ (1 sample: thin line without syrubols), $10^{2}$ ( 2 samples: $0, \bullet$ ), and 10 ( 3 samples: $\square$, $\diamond, \Delta)$. (A) CFU, with the thick line representing batch cultures, (B) PFU

an increase after prolonged incubation. However, both the decrease and the successive increase were comparatively minor. This is in contrast to experiments performed early in this investigation, but in accordance with the findings of the experiment described above.

The extent of phage reproduction in the doublet samples initially inoculated to $10^{2} \mathrm{PFU} \mathrm{ml}^{-1}$ derreased from almost 4 orders of magnitude in the $1 \mathrm{~d}$ samples to little more than 1 order of magnitude in 1 of the $24 \mathrm{~d}$ samples (Fig 4B, middle, open circles). In the $48 \mathrm{~d}$ samples the phage concentration increased again, this time by 5 orders of magnitude, to almost $10^{7} \mathrm{PFU} \mathrm{ml}^{-1}$

Almost perfect synchronism in phage production occurred in the doublet samples coliected after 1, 3 and $48 \mathrm{~d}$. However, for those collected on Days 6, 12, and 24 , the course of phage reproduction differed considerably between the samples (for example see Fig $4 B$, middle, open and filled circles). This was most conspicuous in the $6 \mathrm{~d}$ samples. In one of them, almost no increase in PFU was found 1 d after phage addition, but in the other an increase by a factor of about 250 was found. The final PFU concentrations in this pair differed by a factor of 30 (Table 3)

Similar observations were made with the triplet samples initially containing about $10 \mathrm{PFU} \mathrm{ml}^{-1}$ This is especially true in regard to changes in the extent of phage production. Synchronism was rather weak between samples collected after $1 \mathrm{~d}$ : final PFU concentrations were $1.1 \times 10^{4}, 6.9 \times 10^{4}$, and $3.5 \times 10^{5} \mathrm{PFU}$ $\mathrm{ml}^{-1}$ It was better in the samples collected on Day 3 ; their final PFU values ranged between $2.2 \times 10^{3}$ and $9.9 \times 10^{3}$ PFU mi $\mathrm{ml}^{-1}$ Perfect synchronism was observed with the triplet samples taken on. Day 48; their PFU concentrations during the whole period of observation differed to no greater an extent than that found for their final titres, which ranged between $1.4 \times 10^{6}$ and $2.4 \times 10^{6} \mathrm{PFU} \mathrm{ml}^{-1}$

As with the doublet samples inoculated to initial $10^{2}$ PFU $\mathrm{ml}^{-1}$, considerably weaker synchrony was observed with triplet samples collected after 6, 12, and $24 \mathrm{~d}$. In each of these cases, however, perfect synchronism was found in 2 of the 3 samples, while development in the third sample took another course leading to higher final PFU concentrations (Fig. 4B, middle, triangles).

For the other part of this experiment, 29 samples of $18 \mathrm{ml}$ each were set up in $100 \mathrm{ml}$ Erlenmeyer flasks of known weight. After being placed in the incubator, samples were removed from their places only for phage addition and withdrawal of aliquots for CFU and PFIJ titrations. They remained in place until the end of the experiment, even if they were no longer being used.

A few samples were run as controls, with distilled water or boiled dilution of phage stock to compensate for losses by evaporation. No influence of residual nutrients from phage lysate was observed in the controls replenished with boiled dilution of phage stock. CFU titres for controls never differed significantly from those determined for samples seeded with phage.

At varying times between. 1 and $40 \mathrm{~d}$ of incubation, phage was added to 2 samples each. After $48 \mathrm{~d}$, however, 6 samples were simultaneously seeded with phage. Each time, initial concentrations of $10^{3} \mathrm{PFU}$ $\mathrm{ml}^{-1}$ were generated.

With none of the pairs of samples, except the pair seeded with phage after $4 \mathrm{~d}$ of incubation, was synchronism in phage reproduction observed. For clarity, the results shown in Fig 5 are arranged according to activity of phage reproduction: Fig. 5A shows the results for the sample from each pair. that had lower production, while Fig $5 \mathrm{~B}$ shows results for the more active sample of each pair. Passing reduction in phage reproduction is discernible in both parts of Fig. 5

\section{Pseudolysogeny in bacterial strain $\mathrm{H} 3$}

Results of early tests were inconclusive, not only because of insufficient technique but also due to the unusual reactions of $\mathrm{H} 3$ to phage $\mathrm{H} 3 / 1$ in the employed tests 


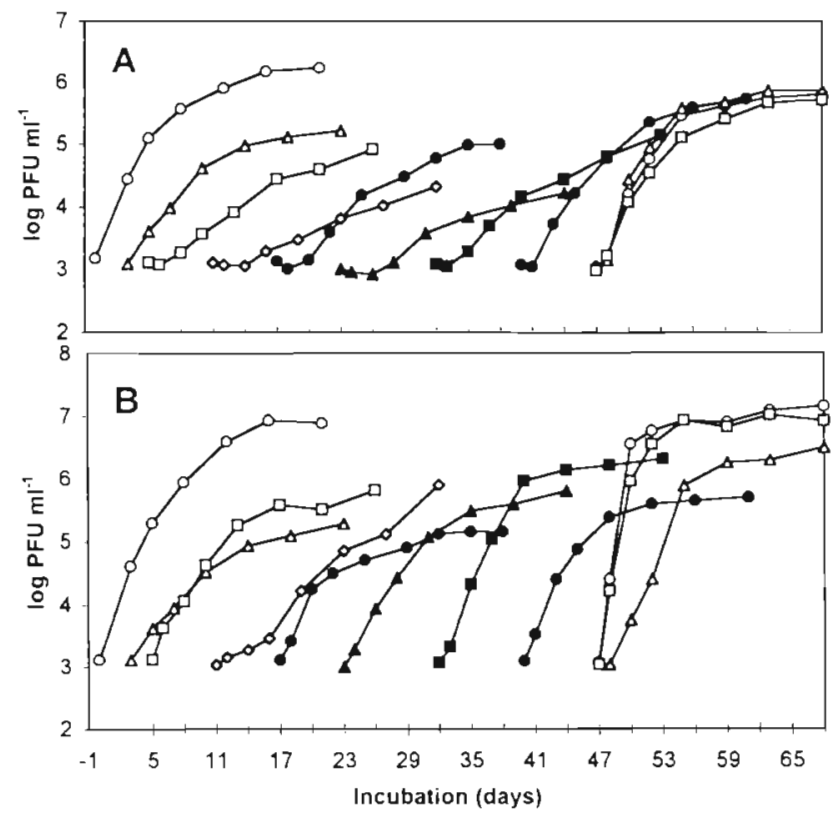

Fig. 5. Individual differences in reproduction of phage $\mathrm{H} 3 / 1$ by host $\mathrm{H} 3$. All samples $(18 \mathrm{ml})$ were set up at the same time (see text) but were inoculated in pairs on different days over the course of the experiment (exception: on Day 47,6 samples were inoculated). (A) and (B) show, respectively, the sample from each pair which had the lower and the higher phage production. Note that only the pair of samples seeded $4 \mathrm{~d}$ after set up showed no difference in phage production

For example, when a suspension of sensitive $\mathrm{H} 3$ cells was cross-streaked over a line of dried-on phage $\mathrm{H} 3 / 1$, lysis of cells was complete within the area of applied phage. A thick wall of growth, however, was often observed at the edge of the area of lysed cells. This wall also occurred with pseudolysogenic material, but more important was the incomplete lysis which occurred within the phage-infested area. Lysis was also sometimes observed within the phage-free part of the area with bacterial growth, though it was incomplete to a lesser degree

These rather confusing observations became intelligible when streaks of bacterial suspensions (about $15 x$ $4 \mathrm{~mm}$ ) were placed on 2 seawater agar (SWA) plates. One plate remained free of phage, the other was prepared for the cross-streak test as usual. It was found to be important to apply phage as uniformly and at as high a concentration as possible.

When the test was sufficiently accurate, the development of pseudolysogeny in $\mathrm{H} 3$ was investigated as follows: From each CFU determination performed in connection with the 6 samples collected from the $200 \mathrm{ml}$ batch culture on Day 85 (the results of which are presented on the right side of Fig. 3), the material of 33 colonies (note that in a few cases more or less than 33 colonies were tested) was suspended in $0.5 \mathrm{ml}$ seawater mixture (SM) each and tested as described above. Only colonies of at least $1.5 \mathrm{~mm}$ in diameter were used. Results representative of all 6 samples tested are compiled in Table 4. The sub-headings ' $<20$ PFU' and ' $>20$ PFU' relate to estimates made from streaks on phage-free SWA, and values in these columns represent instances of 'incomplete' pseudolysogeny, In contrast, streaks containing only scattered colonies account for complete pseudolysogeny. Among more that 2100 tested colonies, only 1 which was genetically resistant was found.

With material from Sample 1 in Table 4 an additional test was performed: From the plates inoculated with the $10^{-2}$ dilution of Sample 1 (see Table 4, Day 25), 12 colonies were cut, each with exactly $1 \mathrm{~cm}^{2}$ of underlying agar, and suspended in $1 \mathrm{ml} \mathrm{SM}$ within the shortest possible time (generally less than 1 min). Each suspension was immediately plated undiluted (PFU and CFU), and a $1 / 10$ dilution for further platings was prepared. The remaining $0.7 \mathrm{ml}$ was mixed with $6.3 \mathrm{ml} \mathrm{SM}$ and this mixture was then filtered through $0.15 \mu \mathrm{m}$ Sartorius filters to remove cells. The filtrates were tested for PFU only. Selected results are presented in Table 5 .

Most of the tested colonies contained high numbers of PFU, and often more PFU than CFU. Between about 30 and $100 \%$ of PFU found with suspensions were detected in filtrates, the percentage increasing with the PFU concentration within a suspension or, respectively, a colony. The number of PFU per colony was inversely correlated with the number of CFU. When the colonies' sizes are taken into account, it can be deduced that a considerable portion of cells present in a tested colony were infected and unable to form colonies after the suspension was plated on SWA.

Finally, the question was addressed as to whether pseudolysogeny of host $\mathrm{H} 3$ was externally induced or whether phage H3/1 was carried for some generations inside the cells without being incorporated into the cells' genomes. It was found that immunity to $\mathrm{H} 3 / 1$ by pseudolysogeny was lost when cell suspensions prepared from pseudolysogenic colonies and appropriately diluted were plated on SWA. When descendant colonies of Colonies $3,8,9,11$, and 12 (see Table 5) were used, pseudolysogeny was detected only in a few descendants of Colony 3. Note that in this case the number of PFU to CFU plated on SWA was about 10 times greater. Of 37 descendant colonies, 15 were detected with complete and 5 with partial pseudolysogeny. The remaining 17 colonies were built from sensitive cells, although at least 2 of them were not completely free of phage $\mathrm{H} 3 / 1$ Irrespective of the phage content of the 37 descendant colonies, only sensitive colonies were found in the generation which followed those 37 
Table 4. Development of pseudolysogeny in phage-host system [H3:H3/1]. Observations made with colonies grown on seawater agar (SWA) within $2 \mathrm{~d}$ at $25^{\circ} \mathrm{C}$ after plating of aliquots collected from samples with increasing phage concentration during incubation (see text). Data are taken from the 6 samples shown on right side of Fig. 3. Complete data are shown here for 2 of the 6 samples, while only final results are shown for the remaining 4. Day: days post inoculation. Incomplete: estimates of plaques observed in a confluent bacterial lawn within the streaks (area about $15 \times 4 \mathrm{~mm}$ ) on a phage-free control plate; on the cross-streak test plate, lysis in phage-seeded area was more or less normal. Complete: non-confluent growth within the streak on the control and test plate. DF: dilution factor of aliquot collected from sample before plating on SWA

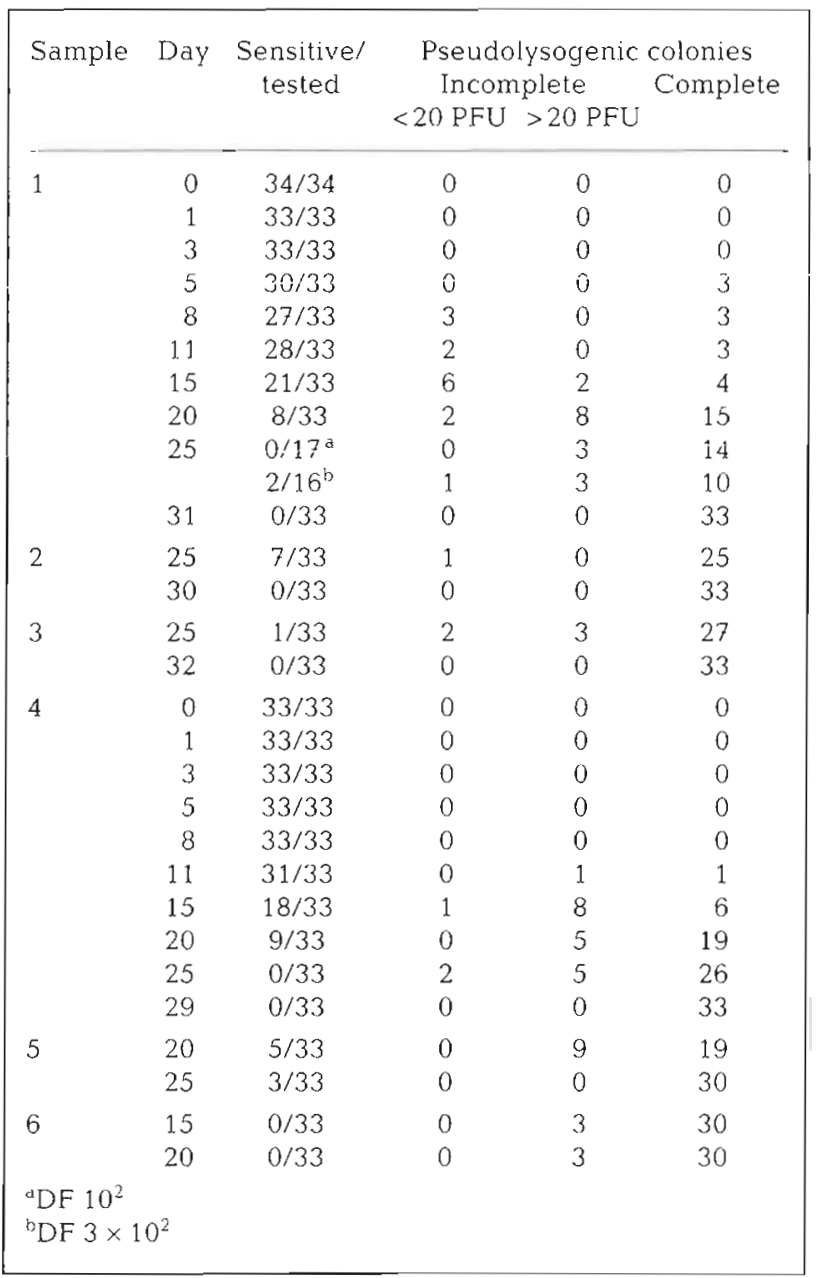

\section{DISCUSSION}

In the preceding paper (Moebus 1996), it was pointed out that problems may arise during an investigation, such as the present one, in which the bacteria used are subcultured many times over a period of more than 2 yr. Varying reactions to essentially similar conditions may be caused by genetically fixed or temporal changes in the physiological state of the bacteria. The latter seems to have been the case in regard to the
Table 5. Pseudolysogeny in phage-host system $|\mathrm{H} 3: \mathrm{H} 3 / 1|$. $\mathrm{CFU}$ and PFU concentrations in suspensions from apparently undamaged colonies grown from a phage-seeded sample (Sample 1: Table 4, Day 25, $10^{-2}$ dilution). Suspensions were prepared in $1 \mathrm{ml}$ seawater mixture, and additional PFU counts were taken from bacteria-free filtrates thereof (see text). Data based on findings with only 1 plate per dilution. s: suspension; $f$ filtrate

\begin{tabular}{|cccccc|}
\hline \multirow{2}{*}{ Col. } & Diam. & \multicolumn{5}{c|}{ Concentration col. } & \\
& $(\mathrm{mm})$ & CFU (s) & PFU (s) & PFU (f) & PFU \\
& & & & \\
1 & 3.1 & $2.2 \times 10^{8}$ & $2.0 \times 10^{8}$ & $6.0 \times 10^{7}$ & 0.3 \\
2 & 1.5 & $2.0 \times 10^{7}$ & $>10^{9}$ & $6.8 \times 10^{8}$ & 0.6 \\
3 & 2.75 & $1.7 \times 10^{8}$ & $>2 \times 10^{9}$ & $>2 \times 10^{9}$ & 1.0 \\
4 & 2.6 & $1.4 \times 10^{8}$ & $>2 \times 10^{9}$ & $>2 \times 10^{9}$ & 1.0 \\
5 & 3.2 & $2.2 \times 10^{8}$ & $>10^{9}$ & $9.0 \times 10^{8}$ & $<1.0$ \\
6 & 2.9 & $1.9 \times 10^{8}$ & $>2 \times 10^{9}$ & $>2 \times 10^{9}$ & 1.0 \\
7 & 3.15 & $1.4 \times 10^{8}$ & $6.2 \times 10^{8}$ & $2.8 \times 10^{8}$ & 0.45 \\
8 & 2.65 & $1.3 \times 10^{8}$ & $4.8 \times 10^{8}$ & $1.4 \times 10^{8}$ & 0.3 \\
9 & 3.15 & $3.6 \times 10^{8}$ & $>2 \times 10^{7}$ & $9.8 \times 10^{6}$ & $<0.5$ \\
10 & 2.8 & $8.3 \times 10^{7}$ & $>10^{9}$ & $>10^{9}$ & 1.0 \\
11 & 3.1 & $>3 \times 10^{8}$ & $6.0 \times 10^{6}$ & $2.5 \times 10^{6}$ & 0.4 \\
12 & 3.1 & $>3 \times 10^{8}$ & $1.4 \times 10^{5}$ & $6.3 \times 10^{4}$ & 0.45 \\
\hline
\end{tabular}

unusual flake formation by host $\mathrm{H} 3$ observed in this study. That it was a temporal change can be concluded from the at least partial return to the 'normal' type reaction observed during the follow-up experiment

The 'normal' reaction of the phage-host system [H3: $\mathrm{H} 3 / 1$ ] is assumed to comprise the changes in rate and extent of phage replication as observed during the first 3 to $4 \mathrm{wk}$ of batch culture. The strong decrease in both parameters repeatedly observed during the first few days after the start of an experiment may be an experimental artifact. Under natural conditions bacteria will meet with abrupt changes in concentrations of nutritive matter; however, it is most probable that this nutritive matter will. rarely be as readily available as the peptone and yeast extract used in this investigation Generally, bacteria will be forced to adapt to ever changing energy sources by producing needed enzymes and/or by activating transport systems.

In contrast, the physiological state attained by recovery in phage production activity, as observed during continued incubation of host $\mathrm{H} 3$, is considered to be ecologically far more relevant. The reason(s) for recovery remain unknown.

Direct counts revealed that $\mathrm{H} 3$ cells adapted to a nutrient level of $0.6 \mathrm{mg}$ YEP $\mathrm{1}^{-1}$ were much smaller than those grown in SWB/5 with a YEP concentration $2000 \times$ higher Furthermore, under the latter condition, less than $10 \%$ of the cells observed were coccoidal. These findings greatly resemble those reported by Novitzky \& Morita (1976). During the present experiments, adapted cells did undergo further changes in size: during incubation of batches, a more or less grad- 
ual increase in the portion of small coccoidal cells (up to $60 \%$ l and a concomitant decrease in rods was observed. Findings for samples tested only at the end of incubation were similar to findings obtained for the respective batch at the time the sample was withdrawn, which indicates that changes in size during incubation of samples, if they even occurred, were probably less pronounced than in batches. This difference between findings from batches and samples was probably due to the fact that the full amount of organic nutrients was available only at the beginning of the experiments.

CFU determinations provided no information pointing to increases in CFU numbers resulting from division to still smaller cells. Even in the experiment impaired by flake formation, samples showed a gradual overall decrease in CFU counts which was similar to that observed with the corresponding batches. Therefore, the newly increased phage propagation is not assumed to be due to an increase in the ratio of sensitive cells to infective phage particles. This statement is supported by the extremely rapid phage production recorded for resting cells, which in no case was preceded or accompanied by the faintest increase in CFU. In contrast, in accordance with such rapid phage production, a slight decrease in CFU numbers was found (Fig, 4), From this, 2 questions arise: (1) What causes such extremely fast increases in the rate of phage production? and (2) Why can most cells survive when they are outnumbered by phage?

What causes such extremely fast increases in the rate of phage production? Based on findings reported for Escherichia coli (Propst-Ricciuti 1972, 1976), the possibility was taken into consideration that starved marine bacteria, when infected by phage, might be unable to release mature progeny virions at once, but instead are able to do so only after an increase in metabolism. No results were obtained which unequivocally support this hypothesis (see also Moebus 1996). For example, $8 \mathrm{~d}$ after inoculation of a sample to about $10 \mathrm{PFU} \mathrm{ml^{-1 }}$ (Fig. 4B, middle, triangles), 5 plaques were found in $0.2 \mathrm{ml}$ withdrawn from that sample, amounting to 25 PFU $\mathrm{mi}^{-1}$. Over the following $4 \mathrm{~d}$ the PFU concentration increased by more than 3 orders of magnitude and during the next $2 \mathrm{~d}$ increased to a final concentration of almost $5 \times 10^{6} \mathrm{PFU} \mathrm{ml}^{-1}$. Such an event may be due to delayed phage release; however, this was a unique observation, and its interpretation as delayed phage release is contradicted by other observations.

In 5 samples collected from a batch incubated for $48 \mathrm{~d}$ and inoculated to an initial $10^{2}$ or $10 \mathrm{PFU} \mathrm{m} \mathrm{m}^{-1}$ (Fig $4 \mathrm{~B}$, right), highly effective phage production started without delay. Such findings were repeatedly obtained with samples withdrawn from batches incubated for the longest period of time in each respective experiment (cf. Figs. 1B, 2 \& 5B) and cannot be explained by delayed phage release.

A first attempt to find an explanation for this phenomenon by using batches of different volume was hampered by strong flake formation during the respective experiment (Fig. 2). In a follow-up experiment (Fig. 4), however, duration of incubation instead of culture volume was found to be the decisive factor.

This does not provide a clue for the basic reason(s) for change in the mode of phage propagation from rather slow and relatively weak, as observed with cells starved for about $3 \mathrm{wk}$, to extremely rapid and effective later on. From results found with identically treated samples such as those presented in Fig. 4 and Table 3 , one is tempted to assume that mere accident is involved. Sometimes an undetected difference in any character of the Erlenmeyer flasks used for samples may have taken effect. That transfer to these receptacles influences the outcome of the experiment was first observed with host $\mathrm{H} 40$ (Moebus 1996), but could also be shown to occur with host H3 (Fig. 3). However, it obviously does not interfere with the time dependent changes in the mode of phage reproduction.

Why can most cells survive when they are outnumbered by phage? Pseudolysogeny was found to cause this phenomenon. Due to varying mechanisms, cells may become immune to the phage in question as long as both partners of the system stay together and the inducing agent is present in sufficient concentration. The nature of the immunizing agent in the case of phage-host system [H3:H3/1] remains unknown; however, it is assumed to be some soluble material released from cells along with progeny phage.

It is possible that pseudolysogeny is rather common among marine bacteria. This assumption by analogous conclusion is based on various observations. The most important are as follows: Firstly, plaques such as those caused by phage $\mathrm{H} 3 / 1$ on $\mathrm{H} 3$ were the plaques most commonly observed in marine PHS isolated from the North Sea and the Atlantic by this author. They consist of a small clear centre, its diameter generally less than $1 \mathrm{~mm}$, and a surrounding zone characterized by microcolonies, the size of which increases with the distance from the plaque's centre. Often, no edge of the plaque can be seen. Such an observation can be attributed to pseudolysogeny induced by material simultaneously released with phage from lysing cells. This material will disperse ahead of the larger virions, causing immunity in uninfected cells. In this way microcolonies within the plaque area must become larger the farther they are from the plaque's centre. The outermost microcolonies will be indistinguishable from the microcolonies forming the bacterial lawn. Secondly, as with many phage strains causing this type of plaque, difficulties were met in producing high-titre phage stocks. 
This was especially true with phages causing plaques with extremely small centres. At first these observations were attributed to the low phage content of such plaques. Therefore, attempts were made to produce phage stock by the use of more than 1 plaque. Neither with the plate elution method nor with liquid cultures were satisfying results obtained. However, the difficulties were overcome by production of phage stock in a 2 -step procedure based on plate elution. In the first step a single plaque, as is usual, is employed to produce a low-titre phage suspension which is then used in a second step leading to high-titre phage stock. This outcome can also be explained by assuming pseudolysogenic reaction, which will be more or less avoided when the majority of cells are infected before the pseudolysogeny-inducing agent reaches critical concentration.

The present results indicate that $\mathrm{H} 3$ cells differ considerably in regard to their ability to develop pseudoIysogeny. If phage is added to bacterial populations up to $1 \mathrm{~d}$ after set up of an experiment, i.e. during exponential growth, most cells will reproduce phage. Under these conditions, the wild-type population generally breaks down and secondary growth of phage-resistant cells occurs, with (sometimes far) less than $1 \%$ of wildtype cells remaining. However, when phage is added at the end of exponential growth, bacterial breakdown may occasionally fail to appear.

This happened in the experiment for which some data are presented in Table 2. For samples collected at the same time from identically treated batches (Batches 3 and 4), PFU concentrations differing by a factor of 15 were found on Day 4 , i.e. 3 d after phage addition. In the first sample from Batch 4 (Sample 1:4), initial phage propagation occurred at a considerably high rate, but ceased completely after $3 \mathrm{~d}$. In the first sample from Batch 3 (Sample 1:3), phage production continued until the end of experiment, leading to a final PFU concentration $4 \times$ higher than that in Sample $1: 4$.

In Sample 1:4, no breakdown of the bacterial population was observed. Obviously, the increase in concentration of the pseudolysogeny-inducing agent resulting from the rapid initial rate of phage reproduction was enough to protect many more cells from phage attack than were protected in Sample 1:3. In this way, however, the extent of phage production in Sample 1:4 was reduced to one fourth.

In samples incubated for more than $1 \mathrm{~d}$ before addition of phage $\mathrm{H} 3 / 1$, breakdown of the $\mathrm{H} 3$ population

This article was submitted to the editor was never found, and colonies of the easily discernible mutant type H3-1 (Moebus 1996) were very rarely encountered. Cells in their stationary phase invariably developed pseudolysogeny independent of the course of phage reproduction. As shown in Fig. 4 (middle), even the extremely rapid increase in PFU concentration in the sample seeded with $10 \mathrm{PFU} \mathrm{ml} \mathrm{ml}^{-1}$ did not cause a steeper decline in CFU than that observed in

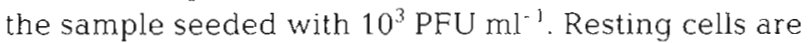
obviously better suited to develop pseudolysogeny than are rapidly growing cells

Whether or not pseudolysogeny develops under natural conditions remains open for speculation. From the available data, one can only conclude that pseudolysogeny would be a highly effective means to protect a dense $\mathrm{H} 3$ population from extinction by phage infection. However, it is very doubtful that a bacterial strain serving as host of a specific phage strain in the water column could attain a population density romparable to those in the samples investigated. As indicated by results reported in the preceding paper (Moebus 1996), competition for nutrients by other bacteria in general would prevent the occurrence of such a situation as well as hinder the production of PFU in numbers as high as were observed in this and the aforementioned investigation.

Acknowledgements. The author is greatly indebted to $\mathrm{Mr}$ $\mathrm{K}$. $\mathrm{W}$. Klings for performing direct counts, taking photographs of specumens and evaluating the results.

\section{LITERATURE CITED}

Baess I (1971) Report on a pseudolysogenic mycobacterium and a review of the literature concerning pseudolysogeny. Acta Pathol Microbiol Scand Sect B Microbiol 79:428-434

Hayes $W(1964)$ The genetics of bacteria and their viruses. Blackwell Scientific Publications, Oxford

Moebus K (1987) Ecology of marine bacteriophages. In: Goyal SM, Gerba CP, Bitton G (eds) Phage ecology. John Wiley \& Sons, New York, p 137-156

Moebus K (1996) Marine bacteriophage reproduction under nutrient-limited growth of host bacteria I. Investigations with six phage-host systems. Mar Ecol Prog Ser 144:1-12

Novitzky JA, Morita RY (1976) Morphological characterization of small cells resulting from nutrient starvation of a psychrophilic marine vibrio. Appl Environ Microbiol 32: $617-622$

Propst-Ricciuti C (1972) Host-virus interactions in Escherichia coli: effect of stationary phase on viral release from MS2infected bacteria. J Virol 10:162-165

Propst-Ricciuti $C$ (1976) The effect of host-cell starvation on virus-induced lysis by MS2 bacteriophage. J Gen Virol 31 $323-330$

Manuscript first received: May 10, 1996

Revised version accepted: July 16, 1996 